\title{
Survival Trends of Right- and Left-Sided Colon Cancer across Four Decades: A Norwegian Population-Based Study
}

Julian Hamfjord ${ }^{1,2,3}$, Tor Åge Myklebust ${ }^{4,5}$, Inger Kristin Larsen ${ }^{4}$, Elin H. Kure ${ }^{2,6}$, Bengt Glimelius ${ }^{7}$, Tormod K. Guren ${ }^{1}$, Kjell M. Tveit ${ }^{3}$, and Marianne G. Guren ${ }^{1}$

\section{ABSTRACT}

Background: Patients with right-sided colon cancer (RCC) and left-sided colon cancer (LCC) differ clinically and molecularly. The main objective was to investigate stage-stratified survival and recurrence of RCC and LCC across four 10-year periods.

Methods: Patients diagnosed from 1977 to 2016 with colon adenocarcinoma were included from the Cancer Registry of Norway. Primary tumor location (PTL) was defined as RCC if proximal and LCC if distal to the splenic flexure. Multivariable regressions were used to estimate HRs for overall survival (OS), recurrence-free survival (RFS), survival after recurrence (SAR), and excess HRs (eHR) for relative survival (RS).

Results: 72,224 patients were eligible for analyses [55.1\% ( $n=$ 39,769/72,224) had RCC]. In 1977 to 1986, there was no difference between LCC and RCC in OS [HR, 1.01; 95\% confidence interval (CI), 0.97-1.06; $P=0.581$ ] or RS (eHR, 0.96; 95\% CI,

\section{Introduction}

Colon cancer is common worldwide (1). In Norway, more than 3,000 cases are diagnosed annually, and the incidence is increasing (2). Right-sided colon cancer (RCC) and left-sided colon cancer (LCC) are today considered to represent biological entities with different molecular, morphologic, and clinical features potentially reflected by different embryologic origin, and different carcinogenetic pathways (3). RCC are more commonly associated with microsatellite instability (MSI), BRAF mutation, and the CpG island methylator phenotype whereas LCC are more frequently chromosomally instable (3). There is

\footnotetext{
${ }^{1}$ Department of Oncology, Oslo University Hospital, Oslo, Norway. ${ }^{2}$ Department of Cancer Genetics, Institute for Cancer Research, Oslo University Hospital, Oslo, Norway. ${ }^{3}$ Institute of Clinical Medicine, Faculty of Medicine, University of Oslo, Oslo, Norway. ${ }^{4}$ Department of Registration, Cancer Registry of Norway, Oslo, Norway. ${ }^{5}$ Department of Research and Innovation, Møre and Romsdal Hospital Trust, Alesund, Norway. ${ }^{6}$ Faculty of Technology, Natural Sciences and Maritime Sciences, University of South-Eastern Norway, Bø in Telemark, Norway. ${ }^{7}$ Department of Immunology, Genetics and Pathology, Uppsala University, Uppsala, Sweden.
}

Note: Supplementary data for this article are available at Cancer Epidemiology, Biomarkers \& Prevention Online (http://cebp.aacrjournals.org/).

Corresponding Author: Marianne G. Guren, Department of Oncology, Oslo University Hospital, P.O. Box 4956 Nydalen, Oslo N-0424, Norway. E-mail: marianne.gronlie.guren@ous-hf.no

Cancer Epidemiol Biomarkers Prev 2022;XX:XX-XX

doi: 10.1158/1055-9965.EPI-21-0555

This open access article is distributed under Creative Commons AttributionNonCommercial-NoDerivatives License 4.0 International (CC BY-NC-ND).

(C)2021 The Authors; Published by the American Association for Cancer Research
$0.90-1.02 ; P=0.179)$. In 2007 to 2016 , LCC had significantly better OS (HR, 0.84; 95\% CI, $0.80-0.87 ; P<0.001)$ and RS (eHR, 0.76 ; $95 \%$ CI, 0.72-0.81; $P<0.001)$ compared with RCC. The gradually diverging and significantly favorable prognosis for LCC was evident for distant disease across all time periods and for regional disease from 2007 onward. There was no difference in RFS between LCC and RCC in patients less than 75 years during 2007 to 2016 (HR, 0.99; 95\% CI, 0.91-1.08; $P=0.819)$; however, SAR was significantly better for LCC (HR, 0.61; 95\% CI, 0.53-0.71; $P<0.001$ ).

Conclusions: A gradually diverging and increasingly favorable prognosis was observed for patients with LCC with advanced disease over the past four decades.

Impact: Current PTL survival disparities stress the need for further exploring targetable molecular subgroups across and within different PTLs to further improve patient outcomes.

a continuum of genetic aberrations from the proximal to distal colon (4). High-grade adenocarcinoma, signet-ring cell carcinoma, and mucinous histology are more frequent in RCC, whereas low-grade adenocarcinomas are more frequent in $\operatorname{LCC}(5,6)$.

At the population level, RCC is associated with older age and more advanced stage at presentation compared with LCC (5). Currently patients with advanced RCC have inferior prognosis compared with LCC, but this may not be valid across all stages and time periods $(7,8)$. In recent trials, patients with metastatic RCC respond more poorly to chemotherapy and anti-EGFR therapy compared with LCC (9). This suggests that primary tumor location (PTL) is a predictor of response to therapy, rather than merely representing a prognostic factor discerning aggressive biology (10).

It is still not clear as to what extent the natural history of RCC differs from patients with LCC, and if the survival disparities observed today can partially be explained by diverging treatment efficacies across time. Based on the available evidence, we postulated that PTL harbors a combination of predictive and prognostic information and hypothesized a trend shift in survival between RCC and LCC across four decades as more effective treatment modalities have gradually been implemented. Hence, the main objective of this study was to investigate the impact of PTL on stage-stratified survival and recurrence across four 10-year periods in the screening-naïve Norwegian population.

\section{Materials and Methods}

\section{Study design and study population}

Data on all colorectal cancer cases diagnosed from 1977 to 2016 were extracted from the Cancer Registry of Norway (CRN). CRN has systematically collected histologic and clinical notifications on all cancer cases in Norway since 1952 and receives death certificates 
from the Cause of Death Registry. The completeness for colon cancer is estimated to be $99.8 \%$ (11). We requested data on diagnosis based on the International Classification of Diseases (ICD), date of diagnosis, sex, age, stage, vital status (alive, deceased, emigrated), and last known vital status date. A subset of patients (2007-2016) had additional variables from the Norwegian Colorectal Cancer Registry including date of first local recurrence and/or metachronous distant metastasis. Confirmed cases of colon adenocarcinomas were included, i.e., other morphologies and rectal cancers were excluded. The study was approved by the CRN and the Data Protection Officer at Oslo University Hospital. Informed consent was waived since the study was strictly register-based.

\section{Morphology}

Morphology was coded according to the ICD for Oncology, Third Edition (ICDO-3), and grouped as adenocarcinomas, mucinous/serous/cystic (MSC) adenocarcinomas, and other carcinomas (Supplementary Table S1).

\section{PTL}

PTL was coded according to the ICD, Revision 7 (ICD-7) and ICDO-3, and grouped as RCC if proximal and LCC if distal to the splenic flexure (Supplementary Table S2). PTL according to ICD-7 was used when evaluating survival trends across four 10-year periods (1977-2016) and ICDO-3 was used when evaluating recurrence during the last 10-year period (2007-2016). Patients with more than one synchronous or metachronous primary cancer were excluded.

\section{Staging}

Stage was coded according to an in-house coding system consistent with the Surveillance, Epidemiology and End Results Program (SEER) Summary staging Manual 2000 (12). In brief, categories were defined as (i) localized if the cancer had not metastasized to lymph nodes or other organs, (ii) regional if the cancer had metastasized to regional lymph nodes and/or had direct extension to surrounding tissues/organs, (iii) distant if the cancer had metastasized to distant lymph nodes or other organs, and (iv) unknown if data could not reliably assert extent of disease. A metastasis was defined as synchronous if diagnosed during the 4 subsequent calendar months after primary diagnosis, and metachronous if diagnosed after this point.

\section{Statistical analyses}

Overall survival (OS) and relative survival (RS) were estimated for patients diagnosed from 1977 to 2016, from time of diagnosis to time of death or censoring (due to emigration, end of follow-up, or administrative censoring at 10 years), whichever occurred first. Recurrence-free survival (RFS) was estimated for patients diagnosed from 2007 to 2016 with localized or regional disease (potentially candidates for surgery with curative intent), from time of diagnosis to time of first recurrence, death, or censoring, whichever occurred first. Survival after recurrence (SAR) was estimated from time of first recurrence to time of death or censoring, whichever occurred first. Complete follow-up on vital status was available until June 30, 2017.

Unadjusted OS and RS curves were estimated using the standard Kaplan-Meier estimator and the Pohar-Perme estimator $(13,14)$, comparing LCC with RCC. Cox proportional hazards regressions were used to estimate HRs for RFS, SAR, and OS. Multivariable flexible parametric regression models were used to model excess mortality due to cancer and to estimate corresponding excess HRs (eHR). A lifetable for the complete Norwegian population, stratified on sex, age (yearly intervals), and calendar year (yearly intervals), provided mortality rates necessary for estimation of RS and eHR. Regressions were adjusted for age group ( $0-49,50-74$, and $\geq 75$ years), sex, stage, and morphology at diagnosis.

RS and excess mortality due to cancer are commonly used as alternatives to analyses of cancer-specific death in situations where one does not trust the information on cause of death provided in the death certificate (15). This may be of importance, particularly for older patients with cancer. As our interest lies in analyzing the effect of PTL, without potential effects of differences in other cause mortality, we did not use competing risk analyses but instead censored individuals at time of other cause death (15).

All analyses were performed using Stata (version 16.1; StataCorp LLC; RRID:SCR_012763; Supplementary Table S3). A $P<0.05$ was considered statistically significant.

\section{Results \\ Study population}

In total, 72,224 patients were eligible for survival analysis [52.6\% $(n=37,984 / 72,224)$ female]. Their median age was 72 years, $55.1 \%$ $(n=39,769 / 72,224)$ had RCC, and 10.0\% $(n=7,193 / 72,224)$ had MSC adenocarcinomas. Stage distribution was $22.2 \%(n=16,013 / 72,224)$ localized, $50.2 \%(n=36,264 / 72,224)$ regional, $24.4 \%(n=17,629 /$ $72,224)$ distant, and $3.2 \%(n=2,318 / 72,224)$ unknown. Over the study period, there was a trend towards older age at time of diagnosis. An increasing proportion of patients were diagnosed with RCC and MSC adenocarcinomas in more recent years. The proportion of regional disease increased whereas localized disease decreased over time, however, the proportion of distant disease was relatively stable (Table 1). A total of $9.0 \%(n=6,474 / 72,224)$ were excluded due to missing PTL, stage, and/or follow-up. The final study populations consisted of 65,750 and 17,796 patients for survival trend and recurrence analysis, respectively (Fig. 1).

\section{Survival}

During the first period (1977-1986), the median OS (mOS) was 3.3 [95\% confidence interval (CI), 3.1-3.4] years for LCC and 3.0 (95\% CI, 2.7-3.2) years for RCC (all stages). The 5 -year OS was $40.9 \%$ for LCC and $40.5 \%$ for RCC (all stages). The 5 -year RS was $50.9 \%$ for LCC and $51.9 \%$ for RCC (all stages). There was no significant difference between LCC and RCC in OS (HR, 1.01; 95\% CI, 0.97-1.06; $P=0.581$ ) or RS (eHR, 0.96; 95\% CI, 0.90-1.02; $P=0.179$ ) when adjusting for age, sex, stage, and morphology (Table 2; Fig. 2A).

During the last period (2007-2016), the mOS was 7.7 (95\% CI, 7.38.1) years for LCC and 6.3 (95\% CI, 6.0-6.6) years for RCC (all stages). The 5-year OS was $59.4 \%$ for LCC and $54.4 \%$ for RCC (all stages). The 5 -year RS was $70.5 \%$ for LCC and $68.2 \%$ for RCC (all stages). LCC was associated with significantly better OS (HR, 0.84; 95\% CI, 0.80 $0.87 ; P<0.001)$ and RS (eHR, 0.76; 95\% CI, 0.72-0.81; $P<0.001$ ) compared with RCC when adjusting for age, sex, stage, and morphology (Table 2; Fig. 2D).

The 5-year RS of colon cancer improved with time for all stages, both sexes, and all age groups. For localized disease, there was no clear difference in survival between LCC and RCC in any time period. For regional disease, LCC had a gradually diverging and significantly more favorable prognosis compared with RCC from 2007 onwards, while for distant disease this trend was evident across all time periods. For patients less than 75 years of age and in men, LCC had a gradually diverging and significantly more favorable 
Table 1. Baseline characteristics of the population eligible for survival analysis.

\begin{tabular}{|c|c|c|c|c|}
\hline & \multicolumn{4}{|c|}{ Population cohort $(n=72,224)$} \\
\hline & $\begin{array}{l}1977-1986 \\
(n=11,935)\end{array}$ & $\begin{array}{l}1987-1996 \\
(n=15,690)\end{array}$ & $\begin{array}{l}1997-2006 \\
(n=19,598)\end{array}$ & $\begin{array}{l}2007-2016 \\
(n=25,001)\end{array}$ \\
\hline \multicolumn{5}{|l|}{ Age } \\
\hline Mean age, years & 69.4 & 70.7 & 71.5 & 71.5 \\
\hline$<50$ years, $n(\%)$ & $665(5.57)$ & $835(5.32)$ & $1,002(5.11)$ & $1,182(4.73)$ \\
\hline 50-74 years, $n(\%)$ & 6,971 (58.41) & 8,368 (53.33) & $9,489(48.42)$ & $12,714(50.85)$ \\
\hline$\geq 75$ years, $n(\%)$ & 4,299 (36.02) & $6,487(41.34)$ & $9,107(46.47)$ & $11,105(44.42)$ \\
\hline \multicolumn{5}{|l|}{ Sex } \\
\hline Female, $n(\%)$ & 6,444 (53.99) & $8,351(53.22)$ & $10,402(53.08)$ & $12,787(51.15)$ \\
\hline Male, $n(\%)$ & $5,491(46.01)$ & 7,339 (46.78) & 9,196 (46.92) & $12,214(48.85)$ \\
\hline \multicolumn{5}{|l|}{ PTL, according to ICD-7 } \\
\hline Right side, $n(\%)$ & 6,096 (51.08) & $8,220(52.39)$ & $11,058(56.42)$ & $14,395(57.58)$ \\
\hline Left side, $n(\%)$ & $5,536(46.38)$ & $6,975(44.46)$ & 7,762 (39.61) & $10,193(40.77)$ \\
\hline Unknown, $n(\%)$ & $303(2.54)$ & $495(3.15)$ & $778(3.97)$ & $413(1.65)$ \\
\hline \multicolumn{5}{|l|}{ PTL, according to ICDO-3 } \\
\hline Right side, $n$ (\%) & - & - & - & $13,546(54.18)$ \\
\hline Left side, $n(\%)$ & - & - & - & $11,042(44.17)$ \\
\hline Unknown, $n(\%)$ & - & - & - & $413(1.65)$ \\
\hline \multicolumn{5}{|l|}{ Morphology, according to ICDO-3 } \\
\hline Adenocarcinomas (814-838), $n$ (\%) & $11,368(95.25)$ & $14,416(91.88)$ & 16,995 (86.72) & $22,102(88.40)$ \\
\hline MSC adenocarcinomas (844-849), $n$ (\%) & $558(4.68)$ & $1,255(8.00)$ & $2,551(13.02)$ & $2,829(11.32)$ \\
\hline Other carcinomas, $n(\%)$ & $9(0.08)$ & $19(0.12)$ & $52(0.27)$ & $70(0.28)$ \\
\hline \multicolumn{5}{|l|}{ Stage } \\
\hline Localized, $n$ (\%) & $3,436(28.79)$ & $4,618(29.43)$ & $3,687(18.81)$ & $4,272(17.09)$ \\
\hline Regional, $n(\%)$ & $5,423(45.44)$ & $6,765(43.12)$ & $10,181(51.95)$ & $13,895(55.58)$ \\
\hline Distant, $n(\%)$ & $2,982(24.99)$ & $3,971(25.31)$ & $4,735(24.16)$ & $5,941(23.76)$ \\
\hline Unknown, $n(\%)$ & $94(0.79)$ & $336(2.14)$ & $995(5.08)$ & $893(3.57)$ \\
\hline \multicolumn{5}{|l|}{ Follow-up } \\
\hline No available follow-up ${ }^{\mathrm{a}}, n(\%)$ & $667(5.59)$ & $708(4.51)$ & $752(3.84)$ & $583(2.33)$ \\
\hline Median follow-up ${ }^{b}$, years & 3.1 & 3.3 & 4.5 & 2.7 \\
\hline Mean follow-up ${ }^{b}$, years & 7.5 & 7.3 & 6.6 & 3.4 \\
\hline
\end{tabular}

${ }^{a}$ Date of diagnosis equals date of death.

${ }^{b}$ Excluding patients with no available follow-up.

prognosis compared with RCC from 1987, while this trend was evident for patients $\geq 75$ years of age and in women from 1997 onwards. For non-MSC adenocarcinomas, LCC had a significantly better prognosis compared with RCC from 1987, while for patients with MSC adenocarcinomas, no clear prognostic trend was observed (Fig. 3; Supplementary Tables S4-S12).

\section{Recurrence}

During the latest period (2007-2016) there was no difference in RFS for LCC compared with RCC in patients $<75$ years of age with localized and regional disease (adjusted HR, 0.99; 95\% CI, 0.91$1.08 ; P=0.819$ ). In contrast, SAR was significantly better for patients with LCC compared with RCC (adjusted HR, 0.61; 95\% CI, 0.53-0.71; $P<0.001$; Fig. 4). The RFS of LCC was borderline inferior to that of RCC in patients $\geq 75$ years of age with localized and regional disease (adjusted HR, 1.07; 95\% CI, 1.00-1.15; $P=$ 0.041). However, SAR was significantly better for LCC compared with RCC also in the elderly population (adjusted HR, 0.80; 95\% CI, $0.68-0.94 ; P=0.005$; Fig. 4).

\section{Discussion}

In this nationwide cohort study we followed more than 65,000 patients diagnosed with colon cancer across four 10-year periods, using high quality and high coverage population data. Several novel trends emerged. Firstly, during 1977 to 1986 there was no overall difference in survival between LCC and RCC, which is supported by scarce historical data suggesting that 5-year survival was close to identical prior to the mid-1980s in the United States (5) and Europe (16). Secondly, from 1987 onwards there has been a gradually diverging and significantly favorable prognosis for LCC, in line with fragmented population- and institution-based observational studies from the United States $(5,7,17)$ and Europe $(6,16)$. A different pattern was identified in patients with MSC adenocarcinomas (mainly mucinous histologies); prognosis of RCC was superior to LCC irrespective of time period, in line with institutional and epidemiologic data previously published $(18,19)$. Distant LCC has rather consistently been associated with better prognosis compared with $\operatorname{RCC}(5,6,20)$. The literature is, however, conflicting with regard to nonmetastatic disease $(21,22)$. A meta-analysis found that patients with LCC had superior prognosis compared with RCC in stage III, whereas the associations were reversed for stage I-II (8). The reasons for discrepancies in individual studies may be due to unaccounted tumor-related factors, patient selection, or inclusion across varying time periods/ treatment regimens. We found an overall shift in survival over the last 40 years favoring LCC, mainly driven by trends in patients below 75 years of age with distant and regional disease and non-MSC morphologies (Fig. 5). We argue that this shift parallels the successive implementation of treatments which have been more efficacious for patients with LCC than RCC; life-prolonging drug therapy for 


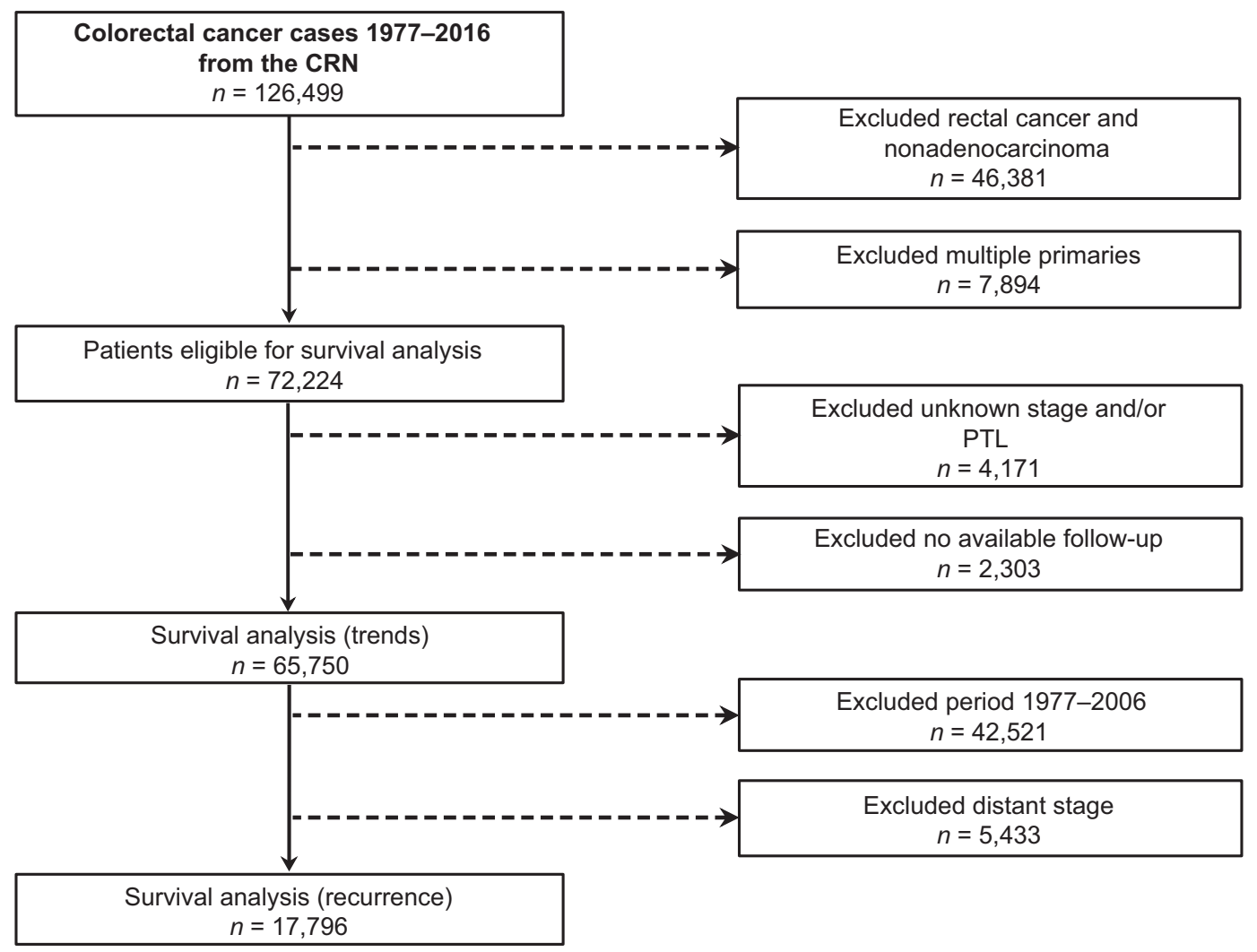

Figure 1.

Overview of data source and extraction, and study populations.

metastatic disease, adjuvant drug therapy for regional disease, and improved surgical interventions of metastases.

Drug therapy for advanced disease was uncommon during the first study period. Yet, prognosis was better for patients with distant LCC. To our knowledge this is a novel finding, suggesting e.g., inherent differences in immunologic control disfavoring advanced/distant RCC (23). The survival gap was further accentuated throughout the following decades. In the 5-fluorouracil monotherapy era until the mid-1990s, no clinical studies prospectively investigated 5-fluorouracil efficacy across PTLs. However, retrospective data from the adjuvant setting suggest that the hypermethylation/hypermutant state of RCC may confer resistance to 5 -fluorouracil $(24,25)$. Later, as combination chemotherapy (5-fluorouracil and irinotecan/oxaliplatin) and targeted therapy were introduced in first-line, meta-analyses confirmed the inferior prognosis of RCC (9). Some results suggest poorer responses of 5-fluorouracil-based combination therapies in patients with RCC (26), but a definite predictive role of PTL in response to combination chemotherapy has not been established $(27,28)$. Regarding targeted therapy, there are indications that the effect of anti-VEGF therapy is independent of PTL (29). Contrary, anti-EGFR therapy has proven more effective in RAS wild-type LCC than RCC $(9,30,31)$. Therapies targeting VEGF and EGFR in first-line were gradually introduced outside of clinical trials from mid-/late-2000s, and treatment was covered for all Norwegian citizens by universal health coverage. Evidence combined; it is plausible that the combined and sequential efficacy of life-prolonging drug therapy has been increasingly biased in favor of LCC across several decades. Molecular variants associated with drug resistance and poor prognosis in advanced disease are far more prevalent on the right (18\% MSI-high; 38\% BRAF-mutated) compared with the left side (1\% MSI-high; 14\% BRAF-mutated) given

Table 2. Unadjusted and adjusted HR and eHR comparing LCC with RCC (reference).

\begin{tabular}{|c|c|c|c|c|c|c|c|c|}
\hline \multirow[b]{3}{*}{ Decade } & \multicolumn{4}{|c|}{ All-cause mortality } & \multicolumn{4}{|c|}{ Excess mortality } \\
\hline & \multicolumn{2}{|c|}{ Unadjusted } & \multicolumn{2}{|c|}{ Adjusted $^{\mathrm{a}}$} & \multicolumn{2}{|c|}{ Unadjusted } & \multicolumn{2}{|c|}{ Adjusted $^{\mathrm{a}}$} \\
\hline & $\overline{\mathrm{HR}(95 \% \mathrm{Cl})}$ & $P$ & $\overline{\mathrm{HR}(95 \% \mathrm{Cl})}$ & $P$ & eHR (95\% Cl) & $P$ & eHR $(95 \% \mathrm{Cl})$ & $P$ \\
\hline 1977-1986 & $0.96(0.92-1.01)$ & 0.089 & $1.01(0.97-1.06)$ & 0.581 & $0.94(0.89-1.00)$ & 0.058 & $0.96(0.90-1.02)$ & 0.179 \\
\hline 1987-1996 & $0.91(0.88-0.95)$ & $<0.001$ & $0.95(0.91-0.99)$ & 0.011 & $0.90(0.85-0.95)$ & $<0.001$ & $0.91(0.86-0.97)$ & 0.002 \\
\hline 1997-2006 & $0.90(0.87-0.93)$ & $<0.001$ & $0.93(0.89-0.96)$ & $<0.001$ & $0.89(0.84-0.94)$ & $<0.001$ & $0.84(0.80-0.89)$ & $<0.001$ \\
\hline 2007-2016 & $0.82(0.79-0.86)$ & $<0.001$ & $0.84(0.80-0.87)$ & $<0.001$ & $0.80(0.75-0.85)$ & $<0.001$ & $0.76(0.72-0.81)$ & $<0.001$ \\
\hline
\end{tabular}

Note: Models are estimated separately for each time period.

${ }^{a}$ Adjusted for age group, sex, stage, and morphology. 
A

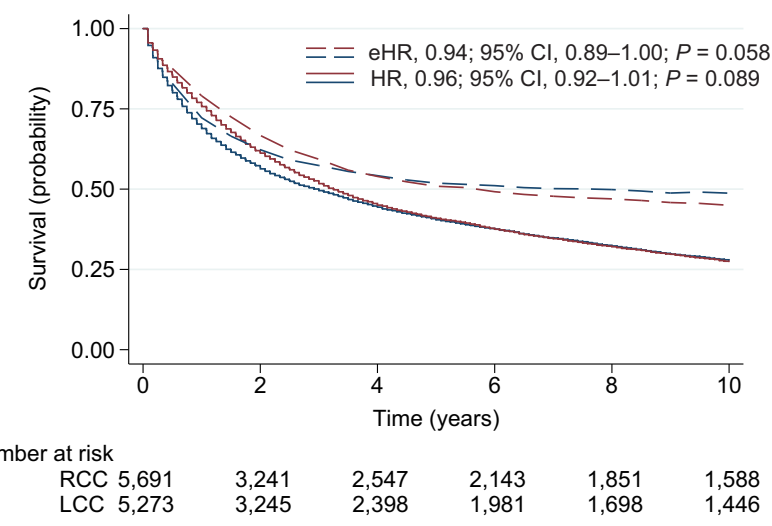

C

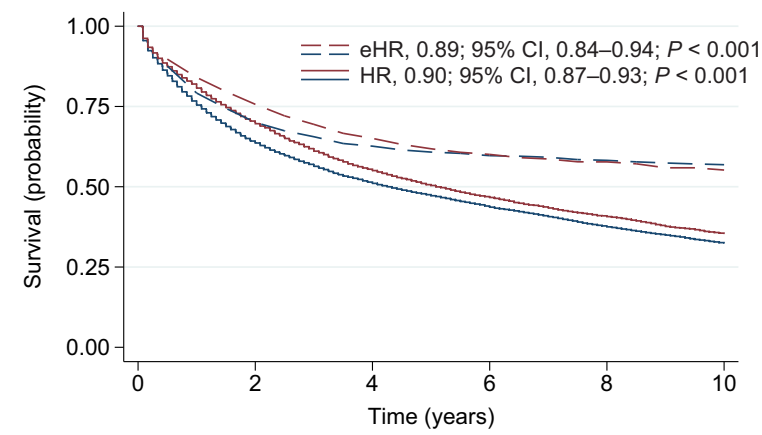

Number at risk

$\begin{array}{llllll}\text { RCC 10,178 } & 6,496 & 5,233 & 4,452 & 3,840 & 3,297 \\ \text { LCC 7,095 } & 4,969 & 3,932 & 3,318 & 2,899 & 2,507\end{array}$

B

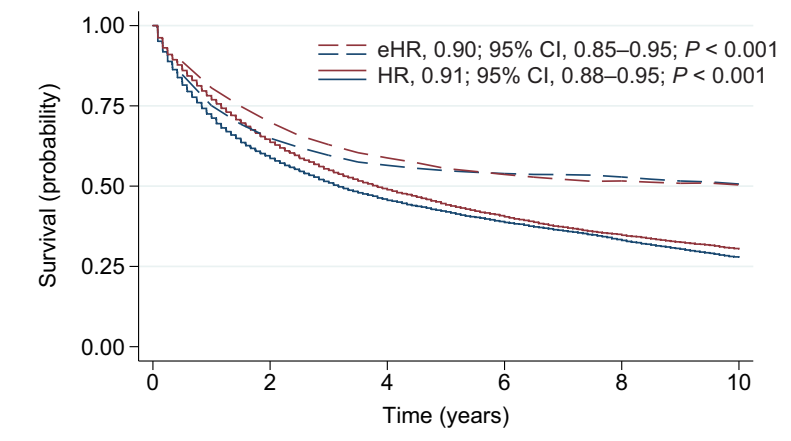

Number at risk

$\begin{array}{llllll}\text { RCC } 7,737 & 4,564 & 3,558 & 3,004 & 2,582 & 2,153 \\ \text { LCC } 6,547 & 4,205 & 3,224 & 2,657 & 2,289 & 1,987\end{array}$

D

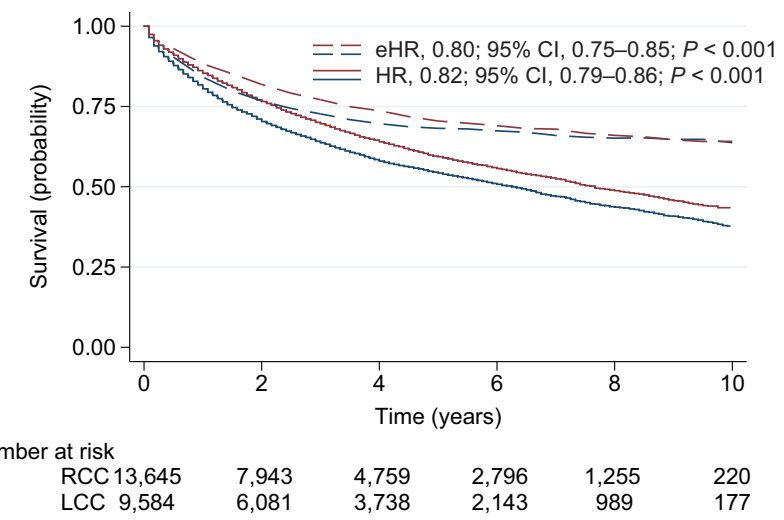

Figure 2.

Survival estimates for all patients stratified by four 10-year periods. Estimates of OS (solid line) and RS (dotted line) are presented for RCC (blue) versus LCC (red) in patients diagnosed during 1977-1986 (A), 1987-1996 (B), 1997-2006 (C), and 2007-2016 (D). HRs and eHRs are unadjusted, comparing LCC with RCC (reference). Numbers at risk are for OS estimates.

real-world data (32), which may account for some of the disparities observed.

Adjuvant drug therapy was introduced as 5-fluorouracil during the mid-/late-1990s with the addition of oxaliplatin from the mid2000s. We found a gradually diverging and significantly favorable prognosis for regional LCC compared with RCC from 2007. Data from the Adjuvant Colon Cancer Endpoints database found that adding oxaliplatin to a 5-flurouracil backbone improved survival more profoundly for LCC than RCC (33), but it is unlikely the sole explanation of the observed trend shift. We therefore investigated RFS and SAR for nonmetastatic patients during the latest study period. There were no major differences in RFS between RCC and LCC, similar to a Swedish population-based study (34). However, SAR was significantly better for patients with LCC. This suggests that today's interventions at time of recurrence (i.e., life-prolonging drug therapy and/or metastasectomy) have major impact on the survival disparity of RCC and LCC. The frequency of patients offered metastasectomy has increased during the last decade, and both metastatic pattern and patient-related factors (e.g., liver-only disease and younger age) favor interventions in patients with LCC $(6,35)$. Consequently, patients treated with e.g., liver resection also differ on the molecular level, with more favorable prognostic characteristics [e.g., 2\% BRAF-mutated in a resected population (36) vs. $19 \% B R A F$-mutated in a real-world metastatic colorectal cancer
(mCRC) population (32)]. Despite this being a highly selected group of patients, a meta-analysis indicated that survival of RCC was inferior to that of LCC after treatment with surgery/ablation of liver metastases (37). Both molecular characteristics and the efficacy of perioperative systemic therapy may be important confounding factors affecting PTL outcome after locoregional treatment, warranting further investigations.

It is likely that PTL represents a proxy of the interplay between molecular (3), immunologic (38), and microbiological factors (39). Patients with RCC more often present with immune cell infiltration, a feature associated with consensus molecular subtype (CMS) 1 and MSI-high (3). Evidence suggests that there is a continuous immunoediting during the evolution of MSI-high colon carcinomas (40). MSIhigh is on one hand associated with higher adaptive immunity, potential for tumor immunosurveillance and equilibrium, and hence prevention of metastatic dissemination (41). On the other hand, MSIhigh has the potential of undergoing immune escape and subsequent disease progression $(42,43)$. Patients with CMS 1/MSI-high have a particularly poor survival after relapse, supporting the hypothesis of immune escape and suggesting poor response to conventional lifeprolonging drug therapy at time of recurrence $(44,45)$. It has also been suggested that the microbiome shape immunesurveillance in RCC affecting efficacy of chemotherapy, programmed cell death protein 1 (PD-1) blockade and overall prognosis (46). A recent study 
Hamfjord et al.

Groups

Overall colon cancer

1977-1986

1987-1996

1997-2006

2007-2016

Stage

Localized

$\begin{array}{ll}\text { 1977-1986 } & 3,208(4.9) \\ 1987-1996 & 4,383(6.7) \\ 1997-2006 & 3,522(5.4) \\ \text { 2007-2016 } & 4,180(6.4) \\ \text { Regional } & \\ 1977-1986 & 5,216(7.9) \\ 1987-1996 & 6,494(9.9) \\ 1997-2006 & 9,642(14.7) \\ 2007-2016 & 13,616(20.7) \\ \text { Distant } & \\ 1977-1986 & 2,540(3.9) \\ 1987-1996 & 3,407(5.2) \\ 1997-2006 & 4,109(6.2) \\ 2007-2016 & 5,433(8.3)\end{array}$

Morphology

Adenocarcinomas

1977-1986

1987-1996

1997-2006

2007-2016

MSC adenocarcinomas

1977-1986

1987-1996

1997-2006

2007-2016

Age

$<75$ years

1977-1986

1987-1996

1997-2006

2007-2016

$\geq 75$ years

1977-1986

1987-1996

1997-2006

2007-2016

Sex

Male

1977-1986

1987-1996

1997-2006

2007-2016

Female

1977-1986

1987-1996

1997-2006

2007-2016

$512(0.8)$

$1,136(1.7)$

2,690 (4.1)

3,766 (5.7)

5,678 (8.6)

5,008 (7.6)

5,956 (9.1)
No. of patients (\%)

$P$ value

$10,964(16.7)$

$14,284(21.7)$

$17,273(26.3)$

$23,229(35.3)$

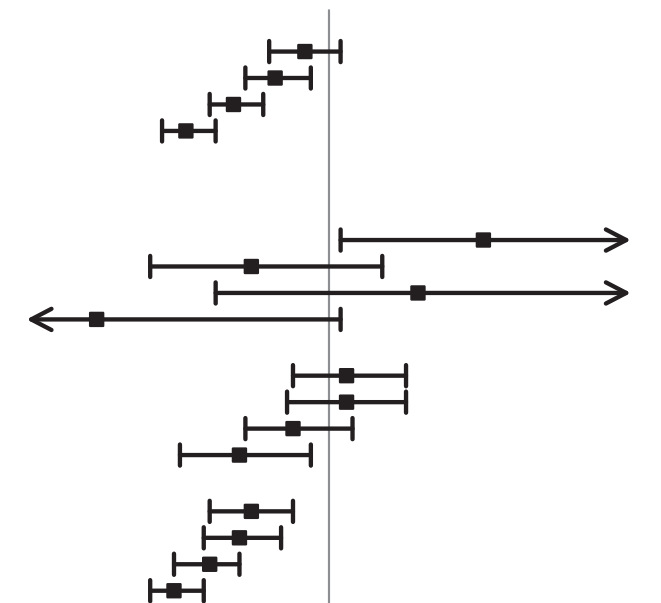

0.179

0.002

$<0.001$

$<0.001$

10,444 (15.9)

$13,130(20.0)$

14,968 (22.8)

20,476 (31.1)

$2,263(3.4)$

$7,198(10.9)$

8,606 (13.1)

$9,499(14.4)$

$13,171(20.0)$

$7,774(11.8)$

10,058 (15.3)

$6,631(10.1)$

8,076 (12.3)

$11,317(17.2)$

$7,653(11.6)$

9,197 (14.0)

11,912 (18.1)

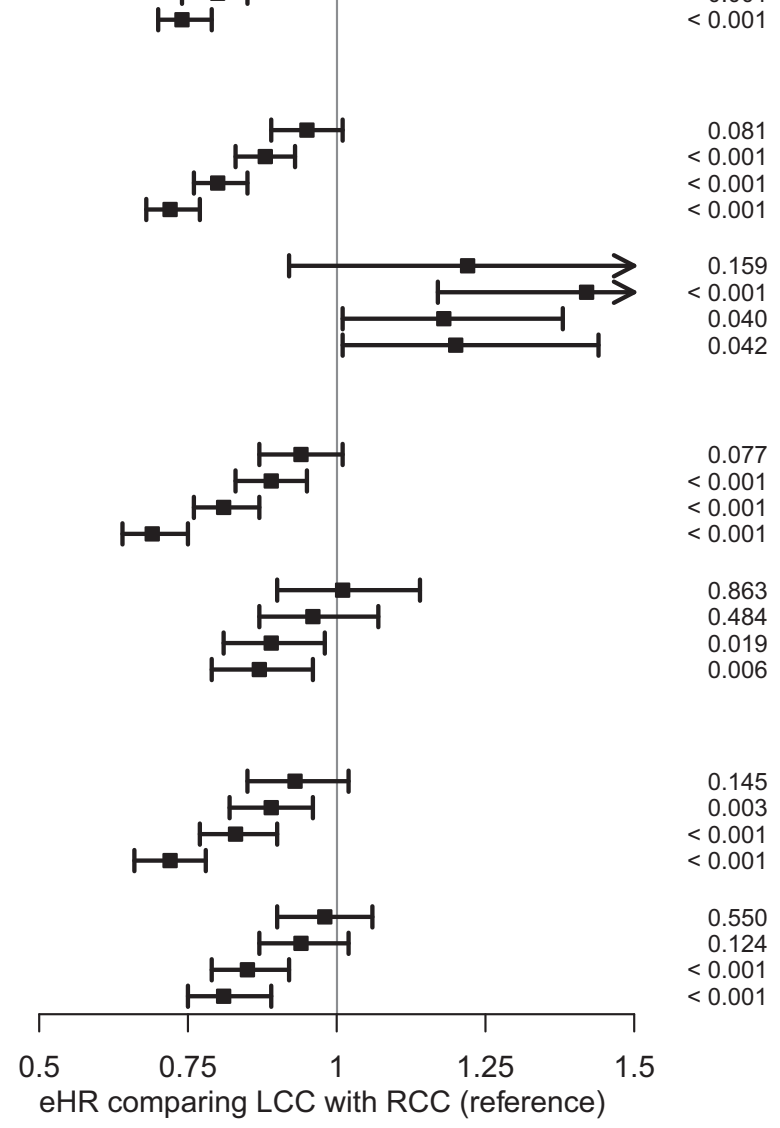

Figure 3.

eHRs associated with PTL according to decades for all colon cancers and different subgroups. Models are estimated separately for each time period and adjusted for age group, sex, stage, and morphology. Percentages may not total 100 due to rounding.

investigating the effects of pembrolizumab or chemotherapy in firstline advanced MSI-high colorectal cancer suggested in subgroup progression-free survival analysis that pembrolizumab was favored in patients with RCC, whereas no significant difference was seen in LCC (47). Thus, we believe there is a need to also consider PTL when designing future treatment trials; and continue investigating the interplay between molecular, immunologic and microbiological fac- tors across and within different anatomic locations and pathological morphologies of the colon.

\section{Strengths}

The predictive and prognostic impact of PTL has previously been examined extensively in the anti-EGFR era. However, we found no nationwide population-based study similar to ours investigating 
A

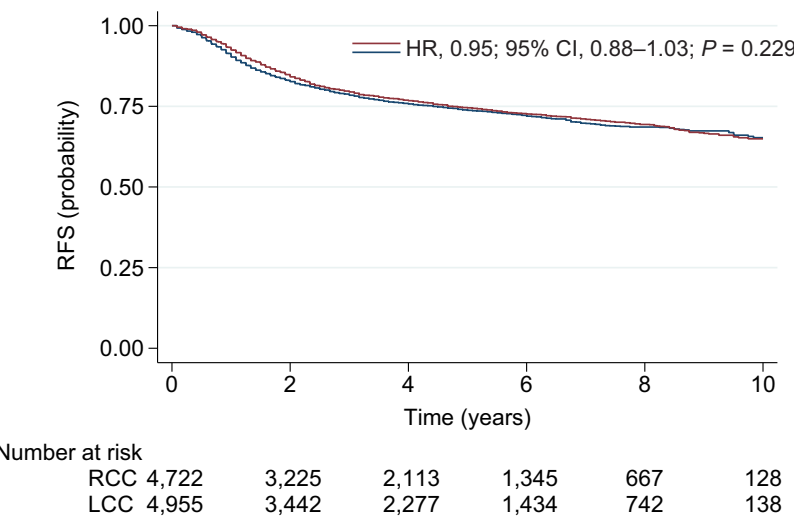

C

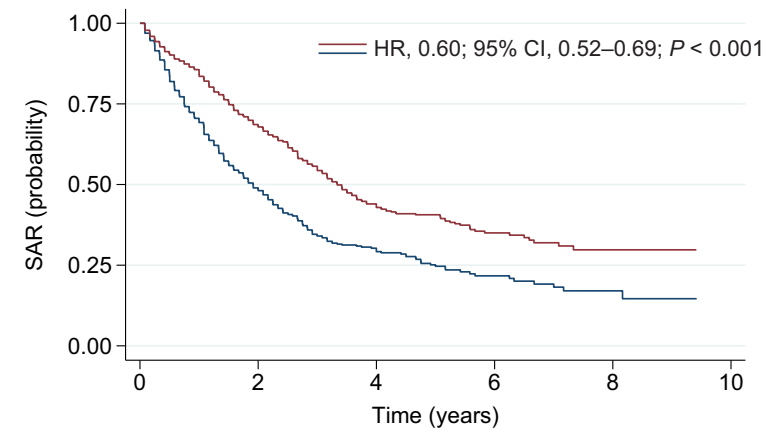

Number at risk

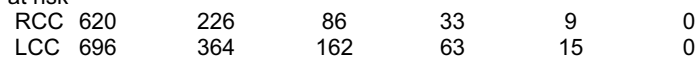

B

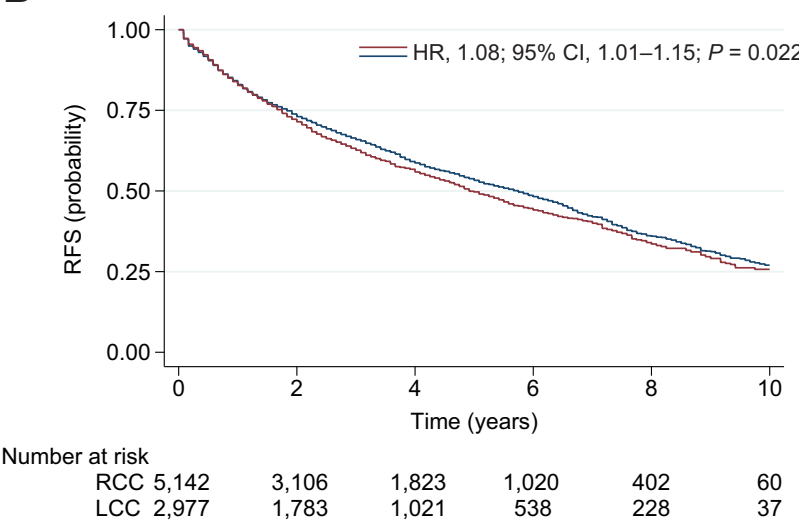

D

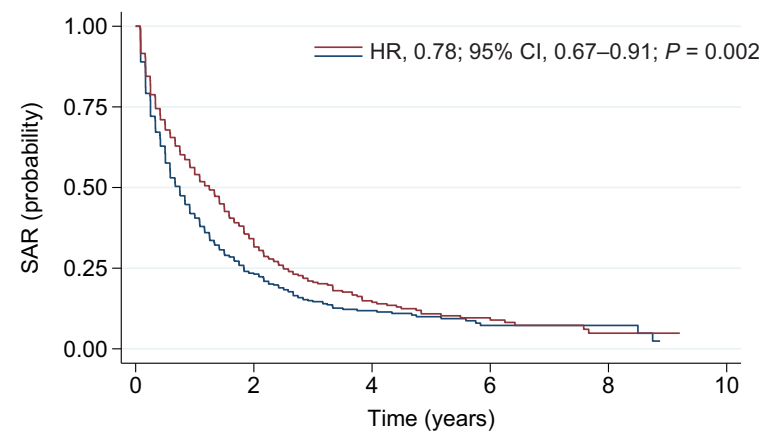

Number at risk

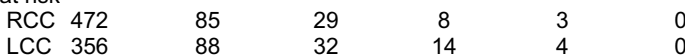

Figure 4.

Recurrence and survival estimates for patients diagnosed with nonmetastatic disease during 2007 to 2016 . RFS is presented for RCC (blue) versus LCC (red) in patients aged 0 to $74(\mathbf{A})$ and $\geq 75$ (B) years. SAR is presented for RCC (blue) versus LCC (red) in patients aged 0 to 74 (C) and $\geq 75$ (D) years. HRs are unadjusted, comparing LCC with RCC (reference).

survival trends across four decades, as more effective treatment modalities have gradually been implemented. Through this study we observed an increasing survival gap disfavoring RCC, which is worrisome and requires attention by the global colorectal cancer community. We believe Norway is considered particularly suitable for this type of investigation. Firstly, Norway has one of the oldest and most complete national cancer registries in the world. Secondly, the Norwegian population is still naive to colorectal cancer screening; no national screening program is yet established, and the effects of conducted pilot studies are expected to be negligible (48). This minimizes the risk that the observed prognostic trends are due to large-scale screening programs, which may be a potential confounder in other population-based studies (49). Finally, universal health coverage was implemented in Norway in the 1950s. This has ensured similar access to advances in oncological treatment for the whole population across decades. This minimizes the risk that the observed prognostic trends are due to inequalities in healthcare access (e.g., variations in private health insurance plans), which may confound other studies.

\section{Limitations}

Firstly, this is an observational study with potential confounders (e.g., comorbidity and molecular data). Secondly, PTL was assigned according to ICD-7 (available across all four 10-year periods) and ICDO-3 (available for the last 10-year period). This shifts PTL about 3\% due to coding of the splenic flexure, but a sensitivity analysis for the last 10-year period revealed comparable results. Thirdly, we observed migration from localized to regional stage, in line with gradually improved pathology services and minor changes in CRN coding rules. Another limitation is coding of regional stage, which includes stage III and some stage II patients. Finally, multiple primaries were pragmatically excluded from the survival analyses $(<10 \%$ of all cases). A sensitivity analysis including one/first primary of patients with multiple primaries revealed comparable results with that of the main analyses.

\section{Conclusion}

In summary, we found a marked trend shift in survival over the past four decades favoring LCC. This was already apparent for distant disease 40 years ago and further accentuated in the following decades; especially for patients below 75 years of age with distant and regional disease, and limited to non-MSC morphologies. We argue that this shift parallels the successive implementation of treatments which are relatively more efficacious for patients with LCC than RCC. We have further shown that RFS is similar for nonmetastatic LCC and RCC, but SAR is significantly inferior for RCC. This substantiates that PTL may 


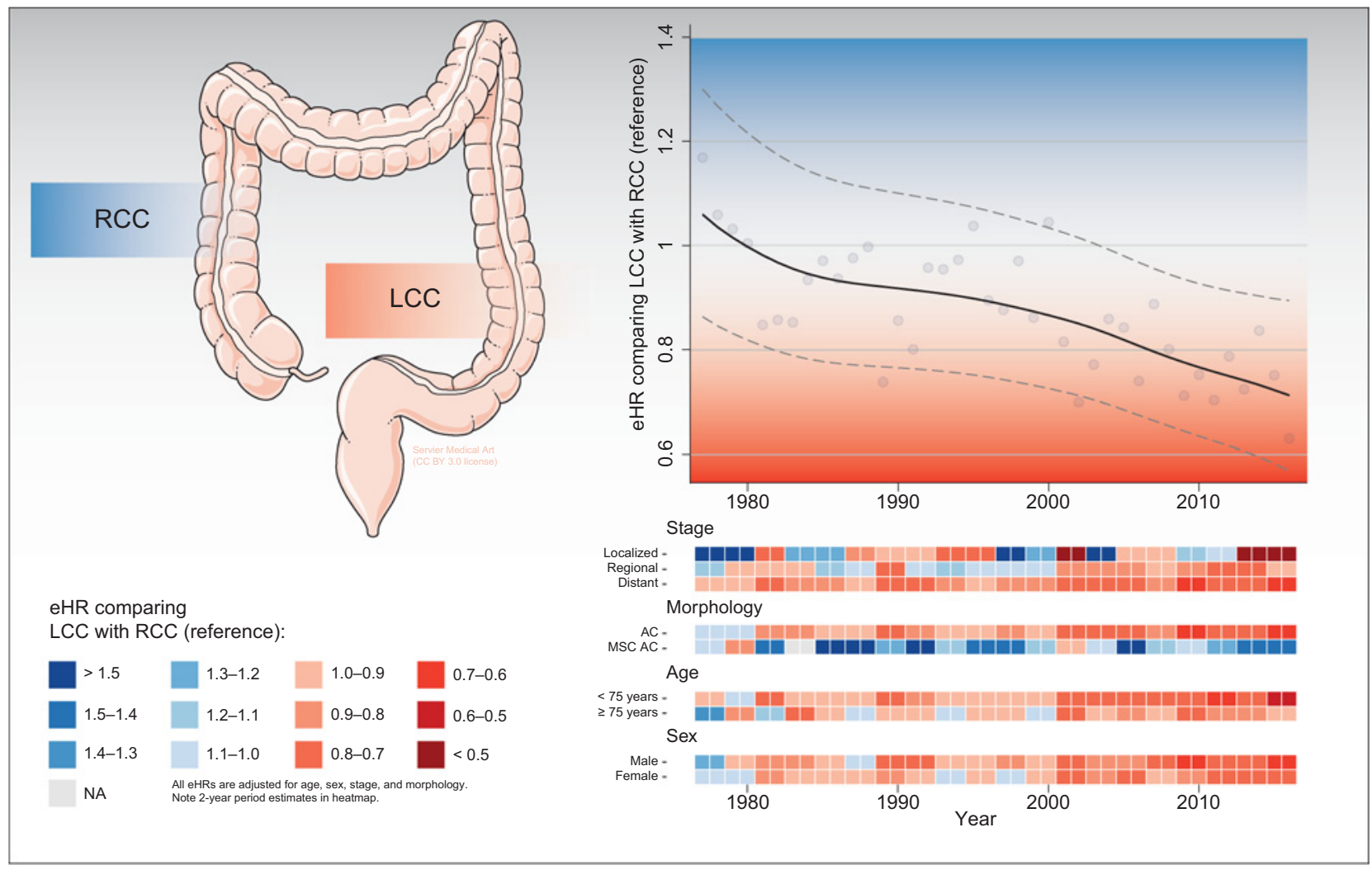

Figure 5.

Graphical abstract summarizing main survival trends comparing LCC with RCC (reference). Top right, the overall eHRs changing over time, including yearly estimates (bullets) and a locally weighted scatterplot smoothing trend line with $95 \% \mathrm{Cls}$. Bottom right, estimated eHR changing over time within different subgroups, including heatmaps stratified for stage, morphology, age, and sex. NA, not available; AC, adenocarcinomas. Medical art in the top left panel was adapted from Servier Medical Art by Servier, licensed under a Creative Commons Attribution 3.0 Unported License.

be predictive of response to systemic therapy in advanced colon cancer, rather than solely being a prognostic marker. We believe there is a need to also consider PTL when designing future treatment trials. Furthermore, dedicated trial protocols should presently be prioritized for patients with MSI-high and BRAF-mutant colon cancer with the aim of closing the survival gap between RCC and LCC.

\section{Authors' Disclosures}

M.G. Guren reports grants from Norwegian Cancer Society during the conduct of the study. No disclosures were reported by the other authors.

\section{Authors' Contributions}

J. Hamfjord: Conceptualization, formal analysis, investigation, visualization, methodology, writing-original draft, writing-review and editing. T.Å. Myklebust: Conceptualization, formal analysis, investigation, visualization, methodology, writing-review and editing. I.K. Larsen: Conceptualization, investigation, methodology, writing-review and editing. E.H. Kure: Investigation, writing-

\section{References}

1. Bray F, Ferlay J, Soerjomataram I, Siegel RL, Torre LA, Jemal A. Global cancer statistics 2018: GLOBOCAN estimates of incidence and mortality worldwide for 36 cancers in 185 countries. CA Cancer J Clin 2018;68:394-424.

2. Araghi M, Soerjomataram I, Bardot A, Ferlay J, Cabasag CJ, Morrison DS, et al. Changes in colorectal cancer incidence in seven high-income countries: a population-based study. Lancet Gastroenterol Hepatol 2019;4:511-8. review and editing. B. Glimelius: Investigation, writing-review and editing T.K. Guren: Investigation, writing-review and editing. K.M. Tveit: Investigation, writing-review and editing. M.G. Guren: Conceptualization, supervision, investigation, methodology, writing-review and editing.

\section{Acknowledgments}

This work was supported by the Norwegian Cancer Society (grant number 190188-2017) to M.G. Guren. The funding organization had no role in study design; the collection, analysis, and interpretation of data; the writing of the report; or in the decision to submit the paper for publication.

The costs of publication of this article were defrayed in part by the payment of page charges. This article must therefore be hereby marked advertisement in accordance with 18 U.S.C. Section 1734 solely to indicate this fact.

Received May 4, 2021; revised July 3, 2021; accepted November 23, 2021 ; published first December 1, 2021

3. Lee MS, Menter DG, Kopetz S. Right versus left colon cancer biology: integrating the consensus molecular subtypes. J Natl Compr Canc Netw 2017;15:411-9.

4. Loree JM, Pereira AAL, Lam M, Willauer AN, Raghav K, Dasari A, et al. Classifying colorectal cancer by tumor location rather than sidedness highlights a continuum in mutation profiles and consensus molecular subtypes. Clin Cancer Res 2018;24:1062-72. 
5. Li Y, Feng Y, Dai W, Li Q, Cai S, Peng J. Prognostic effect of tumor sidedness in colorectal cancer: a SEER-based analysis. Clin Colorectal Cancer 2019;18: e104-e16.

6. Brouwer NPM, van der Kruijssen DEW, Hugen N, de Hingh I, Nagtegaal ID, Verhoeven RHA, et al. The impact of primary tumor location in synchronous metastatic colorectal cancer: differences in metastatic sites and survival. Ann Surg Oncol 2020;27:1580-8.

7. Wang CB, Shahjehan F, Merchea A, Li Z, Bekaii-Saab TS, Grothey A, et al. Impact of tumor location and variables associated with overall survival in patients with colorectal cancer: a mayo clinic colon and rectal cancer registry study. Front Oncol 2019;9:76.

8. Ha GW, Kim JH, Lee MR. Oncologic effects of primary tumor-sidedness on patients with stages 1-3 colon cancer: a meta-analysis. Ann Surg Oncol 2019;26: 1366-75.

9. Holch JW, Ricard I, Stintzing S, Modest DP, Heinemann V. The relevance of primary tumour location in patients with metastatic colorectal cancer: a meta-analysis of first-line clinical trials. Eur J Cancer 2017;70:87-98.

10. Kerr DJ, Domingo E, Kerr R. Is sidedness prognostically important across all stages of colorectal cancer? Lancet Oncol 2016;17:1480-2.

11. Larsen IK, Smastuen M, Johannesen TB, Langmark F, Parkin DM, Bray F, et al. Data quality at the Cancer Registry of Norway: an overview of comparability, completeness, validity and timeliness. Eur J Cancer 2009; 45:1218-31.

12. Walters S, Maringe C, Butler J, Brierley JD, Rachet B, Coleman MP Comparability of stage data in cancer registries in six countries: lessons from the International Cancer Benchmarking Partnership. Int J Cancer 2013 132:676-85.

13. Perme MP, Stare J, Esteve J. On estimation in relative survival. Biometrics 2012; 68:113-20.

14. Roche L, Danieli C, Belot A, Grosclaude P, Bouvier AM, Velten M, et al Cancer net survival on registry data: use of the new unbiased Pohar-Perme estimator and magnitude of the bias with the classical methods. Int J Cancer 2013;132:2359-69.

15. Eloranta S, Smedby KE, Dickman PW, Andersson TM. Cancer survival statistics for patients and healthcare professionals - a tutorial of real-world data analysis. J Intern Med 2021;289:12-28.

16. Gervaz P, Usel M, Rapiti E, Chappuis P, Neyroud-Kaspar I, Bouchardy C. Right colon cancer: left behind. Eur J Surg Oncol 2016;42:1343-9.

17. Meguid RA, Slidell MB, Wolfgang CL, Chang DC, Ahuja N. Is there a difference in survival between right- versus left-sided colon cancers? Ann Surg Oncol 2008; 15:2388-94.

18. Ishihara S, Watanabe T, Akahane T, Shimada R, Horiuchi A, Shibuya H, et al. Tumor location is a prognostic factor in poorly differentiated adenocarcinoma mucinous adenocarcinoma, and signet-ring cell carcinoma of the colon. Int J Colorectal Dis 2012;27:371-9.

19. Qiu MZ, Pan WT, Lin JZ, Wang ZX, Pan ZZ, Wang FH, et al. Comparison of survival between right-sided and left-sided colon cancer in different situations. Cancer Med 2018;7:1141-50

20. Wang C, Wainberg ZA, Raldow A, Lee P. Differences in cancer-specific mortality of right- versus left-sided colon adenocarcinoma: a surveillance, epidemiology, and end results database analysis. JCO Clin Cancer Inform 2017;1:1-9.

21. Petrelli F, Tomasello G, Borgonovo K, Ghidini M, Turati L, Dallera P, et al. Prognostic survival associated with left-sided vs right-sided colon cancer: a systematic review and meta-analysis. JAMA Oncol 2017;3:211-9.

22. Karim S, Brennan K, Nanji S, Berry SR, Booth CM. Association between prognosis and tumor laterality in early-stage colon cancer. JAMA Onco 2017;3:1386-92

23. Dunn GP, Bruce AT, Ikeda H, Old LJ, Schreiber RD. Cancer immunoediting: from immunosurveillance to tumor escape. Nat Immunol 2002;3:991-8.

24. Sargent DJ, Marsoni S, Monges G, Thibodeau SN, Labianca R, Hamilton SR, et al. Defective mismatch repair as a predictive marker for lack of efficacy of fluorouracil-based adjuvant therapy in colon cancer. J Clin Oncol 2010;28: 3219-26.

25. Ribic CM, Sargent DJ, Moore MJ, Thibodeau SN, French AJ, Goldberg RM, et al. Tumor microsatellite-instability status as a predictor of benefit from fluorouracil-based adjuvant chemotherapy for colon cancer. N Engl J Med 2003;349:247-57.
26. Modest DP, Schulz C, von Weikersthal LF, Quietzsch D, von Einem JC Schalhorn A, et al. Outcome of patients with metastatic colorectal cancer depends on the primary tumor site (midgut vs. hindgut): analysis of the FIRE1-trial (FuFIRI or mIROX as first-line treatment). Anticancer Drugs 2014;25:212-8.

27. Seligmann JF, Elliott F, Richman SD, Southward K, Barrett J, Quirke P, et al Primary tumour location $(\mathrm{Ptl})$ as a prognostic and predictive factor in advanced colorectal cancer (Acrc): data from 2075 patients (Pts) in randomised trials Ann Oncol 2014;25:iv172.

28. Ochiai T, Nishimura K, Watanabe T, Kitajima M, Nakatani A, Nagayasu K, et al Impact of primary tumor location as a predictive factor in patients suffering from colorectal cancer treated with cytotoxic anticancer agents based on the collagen gel droplet-embedded drug sensitivity test. Oncol Lett 2019;17:1842-50.

29. Loupakis F, Hurwitz HI, Saltz L, Arnold D, Grothey A, Nguyen QL, et al. Impact of primary tumour location on efficacy of bevacizumab plus chemotherapy in metastatic colorectal cancer. Br J Cancer 2018;119:1451-5.

30. Arnold D, Lueza B, Douillard JY, Peeters M, Lenz HJ, Venook A, et al. Prognostic and predictive value of primary tumour side in patients with RAS wild-type metastatic colorectal cancer treated with chemotherapy and EGFR directed antibodies in six randomized trials. Ann Oncol 2017;28:1713-29.

31. Tejpar S, Stintzing S, Ciardiello F, Tabernero J, Van Cutsem E, Beier F, et al Prognostic and predictive relevance of primary tumor location in patients with RAS wild-type metastatic colorectal cancer: retrospective analyses of the CRYS TAL and FIRE-3 trials. JAMA Oncol 2017;3:194-201.

32. Nunes L, Aasebo K, Mathot L, Ljungstrom V, Edqvist PH, Sundstrom M, et al Molecular characterization of a large unselected cohort of metastatic colorectal cancers in relation to primary tumor location, rare metastatic sites and prognosis. Acta Oncol 2020;59:417-26.

33. Salem ME, Yin J, Goldberg RM, Pederson LD, Wolmark N, Alberts SR, et al Evaluation of the change of outcomes over a 10-year period in patients with stage III colon cancer: pooled analysis of 6501 patients treated with fluorouracil, leucovorin, and oxaliplatin in the ACCENT database. Ann Oncol 2020;31:480-6.

34. Osterman E, Glimelius B. Recurrence risk after up-to-date colon cancer staging, surgery, and pathology: analysis of the entire Swedish population. Dis Colon Rectum 2018;61:1016-25.

35. Siebenhuner AR, Guller U, Warschkow R. Population-based SEER analysis of survival in colorectal cancer patients with or without resection of lung and liver metastases. BMC Cancer 2020;20:246.

36. Gagniere J, Dupre A, Gholami SS, Pezet D, Boerner T, Gonen M, et al. Is hepatectomy justified for BRAF mutant colorectal liver metastases?: A multiinstitutional analysis of 1497 patients. Ann Surg 2020;271:147-54.

37. Liu W, Wang HW, Wang K, Xing BC. The primary tumor location impacts survival outcome of colorectal liver metastases after hepatic resection: a systematic review and meta-analysis. Eur J Surg Oncol 2019; 45:1349-56.

38. Angell HK, Bruni D, Barrett JC, Herbst R, Galon J. The immunoscore: colon cancer and beyond. Clin Cancer Res 2020;26:332-9.

39. Ternes D, Karta J, Tsenkova M, Wilmes P, Haan S, Letellier E. Microbiome in colorectal cancer: how to get from meta-omics to mechanism? Trends Microbio 2020;28:401-23

40. Ballhausen A, Przybilla MJ, Jendrusch M, Haupt S, Pfaffendorf E, Seidler F, et al. The shared frameshift mutation landscape of microsatellite-unstable cancers suggests immunoediting during tumor evolution. Nat Commun 2020;11:4740.

41. Mlecnik B, Bindea G, Angell HK, Maby P, Angelova M, Tougeron D, et al Integrative analyses of colorectal cancer show immunoscore is a stronger predictor of patient survival than microsatellite instability. Immunity 2016 ; 44:698-711.

42. Grasso CS, Giannakis M, Wells DK, Hamada T, Mu XJ, Quist M, et al Genetic mechanisms of immune evasion in colorectal cancer. Cancer Discov 2018;8:730-49.

43. Mlecnik B, Bindea G, Kirilovsky A, Angell HK, Obenauf AC, Tosolini M, et al The tumor microenvironment and Immunoscore are critical determinants of dissemination to distant metastasis. Sci Transl Med 2016;8:327ra26.

44. Guinney J, Dienstmann R, Wang X, de Reynies A, Schlicker A, Soneson C et al. The consensus molecular subtypes of colorectal cancer. Nat Med 2015 21:1350-6.

45. Kim CG, Ahn JB, Jung M, Beom SH, Kim C, Kim JH, et al. Effects of microsatellite instability on recurrence patterns and outcomes in colorectal cancers. Br J Cancer 2016;115:25-33. 
Hamfjord et al.

46. Roberti MP, Yonekura S, Duong CPM, Picard M, Ferrere G, Tidjani Alou M, et al. Chemotherapy-induced ileal crypt apoptosis and the ileal microbiome shape immunosurveillance and prognosis of proximal colon cancer. Nat Med 2020;26: 919-31.

47. Andre T, Shiu KK, Kim TW, Jensen BV, Jensen LH, Punt C, et al. Pembrolizumab in microsatellite-instability-high advanced colorectal cancer. N Engl J Med 2020; 383:2207-18.
48. Holme O, Schoen RE, Senore C, Segnan N, Hoff G, Loberg M, et al. Effectiveness of flexible sigmoidoscopy screening in men and women and different age groups: pooled analysis of randomised trials. BMJ 2017;356:16673.

49. Cardoso R, Guo F, Heisser T, Hackl M, Ihle P, De Schutter H, et al. Colorectal cancer incidence, mortality, and stage distribution in European countries in the colorectal cancer screening era: an international population-based study. Lancet Oncol 2021;22:1002-13. 


\section{Cancer Epidemiology, \\ Biomarkers \& Prevention}

\section{Survival Trends of Right- and Left-Sided Colon Cancer across Four Decades: A Norwegian Population-Based Study} Julian Hamfjord, Tor Åge Myklebust, Inger Kristin Larsen, et al.

Cancer Epidemiol Biomarkers Prev Published OnlineFirst December 1, 2021.

\section{Updated version Access the most recent version of this article at: doi:10.1158/1055-9965.EPI-21-0555}

Supplementary Access the most recent supplemental material at: Material http://cebp.aacrjournals.org/content/suppl/2021/12/01/1055-9965.EPI-21-0555.DC1

E-mail alerts Sign up to receive free email-alerts related to this article or journal.

Reprints and To order reprints of this article or to subscribe to the journal, contact the AACR Publications Subscriptions Department at pubs@aacr.org.

Permissions To request permission to re-use all or part of this article, use this link http://cebp.aacrjournals.org/content/early/2021/12/22/1055-9965.EPI-21-0555.

Click on "Request Permissions" which will take you to the Copyright Clearance Center's (CCC)

Rightslink site. 